\title{
Time-varying effects of screen media exposure in the relationship between socioeconomic background and childhood obesity: eAppendices
}

\section{eAppendix 1. Missings}

eTable A1.1. Percent missings.

\begin{tabular}{|c|c|}
\hline Variable & Percent missing \\
\hline Maternal psychological distress (age 7) & 13 \\
\hline Maternal psychological distress (age 5) & 11 \\
\hline Income (age 7) & 10 \\
\hline BMI (age 7) & 9 \\
\hline Mother's education & 9 \\
\hline Not enough time to spend with child (age 7) & 9 \\
\hline Managing financially (age 7) & 9 \\
\hline Housing tenure (age 7) & 9 \\
\hline Screen media exposure (age 7) & 9 \\
\hline Maternal fair/poor self-rated health (age 7) & 9 \\
\hline Area deprivation (age 7) & 8 \\
\hline Child illness that limits activity (age 7) & 8 \\
\hline Child attends club outside of school (age 7) & 8 \\
\hline Parent(s) not in work (age 7) & 8 \\
\hline Number of parents/carers sweep4 & 8 \\
\hline Natural father in household (age 7) & 8 \\
\hline Number of siblings (age 7) & 8 \\
\hline Maternal BMI (age 7) & 8 \\
\hline BMI (age 5) & 7 \\
\hline Income (age 5) & 7 \\
\hline Not enough time to spend with child (age 5) & 6 \\
\hline Obesity & 6 \\
\hline Managing financially (age 5) & 6 \\
\hline Housing tenure (age 5) & 6 \\
\hline Maternal fair/poor self-rated health (age 5) & 6 \\
\hline Parent(s) not in work (age 5) & 6 \\
\hline Screen media exposure (age 11) & 6 \\
\hline Child illness that limits activity (age 5) & 6 \\
\hline Child attends club outside of school (age 5) & 6 \\
\hline Number of parents/carers (age 5) & 6 \\
\hline Area deprivation (age 5) & 6 \\
\hline Natural father in household (age 5) & 6 \\
\hline Number of siblings (age 5) & 6 \\
\hline Maternal BMI (age 5) & 6 \\
\hline Mother's cognitive ability & 6 \\
\hline Ethnicity & 1 \\
\hline Mother’s religion & 0 \\
\hline Mother's age at birth & 0 \\
\hline Age & 0 \\
\hline Gender & 0 \\
\hline Country & 0 \\
\hline
\end{tabular}




\section{eAppendix 2. Interaction effects}

eTable A2.1. Results from the inverse-probability-weighted regression model regressing obesity on educational level and screen media exposure, including interaction terms between educational level and screen media exposure at age 7 .

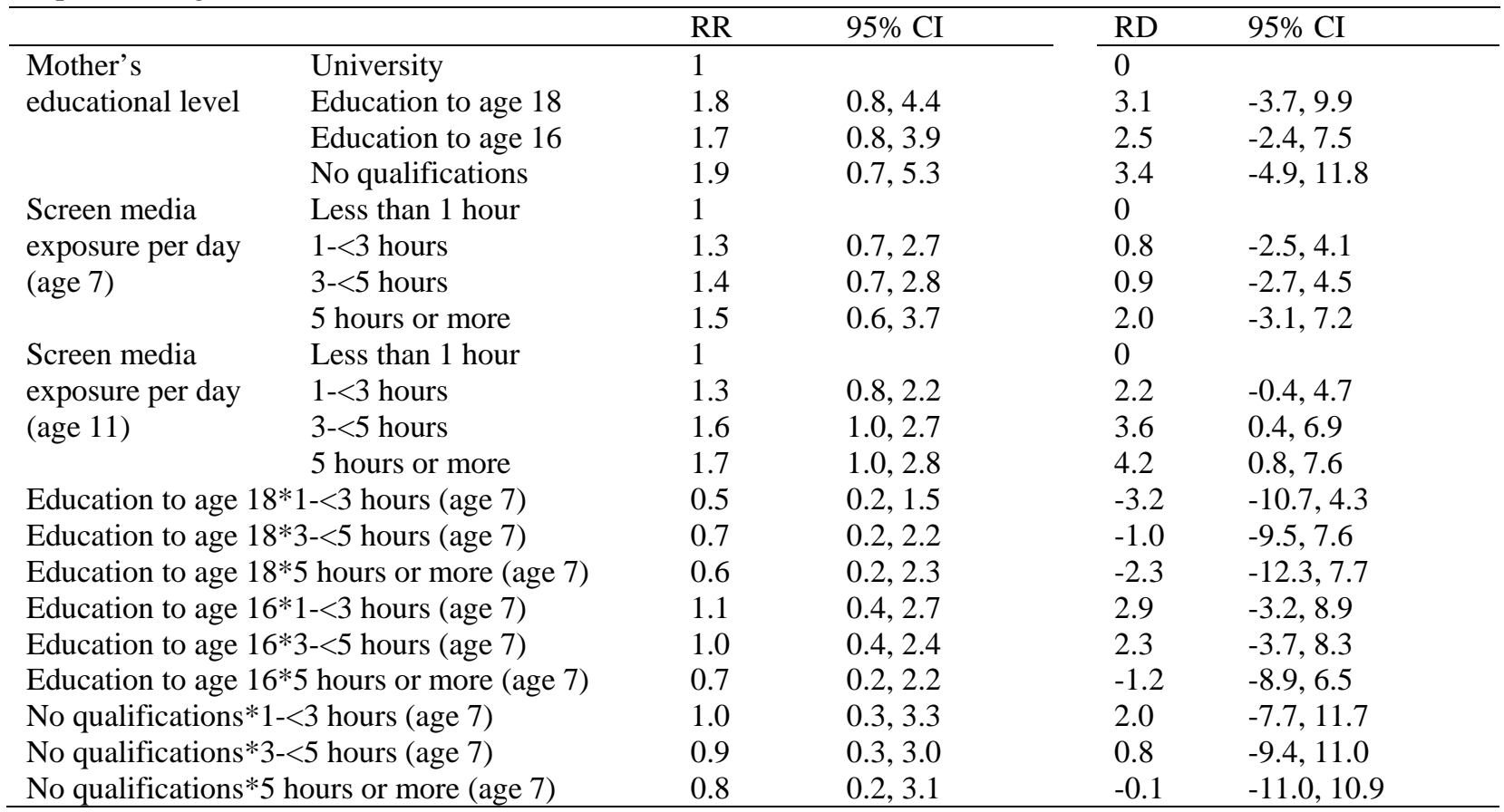

F-test of the cross-product terms on the risk ratio scale: $F(9,1000)=0.43 ; \mathrm{p}=0.920$

F-test of the cross-product terms on the risk difference scale: $F(9,1000)=0.45 ; p=0.905$

eTable A2.2. Results from the inverse-probability-weighted regression model regressing obesity on educational level and screen media exposure, including interaction terms between educational level and screen media exposure at age 11 .

\begin{tabular}{|c|c|c|c|c|c|}
\hline \multirow{3}{*}{$\begin{array}{l}\text { Mother's } \\
\text { educational level }\end{array}$} & \multirow[b]{2}{*}{ University } & \multirow{2}{*}{$\frac{\mathrm{RR}}{1}$} & \multirow[t]{2}{*}{$95 \%$ CI } & \multirow{2}{*}{$\begin{array}{l}\text { RD } \\
0\end{array}$} & \multirow[t]{2}{*}{$95 \% \mathrm{CI}$} \\
\hline & & & & & \\
\hline & Education to age 18 & 1.3 & $0.3,6.1$ & 1.6 & $-8.1,11.4$ \\
\hline & Education to age 16 & 2.3 & $0.9,6.4$ & 4.2 & $-2.2,10.6$ \\
\hline & No qualifications & 1.9 & $0.4,8.3$ & 2.8 & $-6.3,11.9$ \\
\hline \multirow{4}{*}{$\begin{array}{l}\text { Screen media } \\
\text { exposure per day } \\
\text { (age } 7 \text { ) }\end{array}$} & Less than 1 hour & 1 & & 0 & \\
\hline & $1-<3$ hours & 1.3 & $0.9,1.9$ & 1.4 & $-1.0,3.8$ \\
\hline & $3-<5$ hours & 1.3 & $0.8,1.9$ & 1.6 & $-1.0,4.2$ \\
\hline & 5 hours or more & 1.2 & $0.7,1.8$ & 1.1 & $-1.9,4.1$ \\
\hline \multirow{4}{*}{$\begin{array}{l}\text { Screen media } \\
\text { exposure per day } \\
\text { (age } 11 \text { ) }\end{array}$} & Less than 1 hour & 1 & & 0 & \\
\hline & $1-<3$ hours & 1.7 & $0.7,3.8$ & 2.0 & $-1.4,5.3$ \\
\hline & $3-<5$ hours & 1.7 & $0.7,4.3$ & 1.9 & $-2.6,6.4$ \\
\hline & 5 hours or more & 1.9 & $0.8,4.8$ & 3.0 & $-1.6,7.5$ \\
\hline \multicolumn{2}{|c|}{ Education to age $18 * 1-<3$ hours (age 11 ) } & 0.8 & $0.2,4.0$ & -0.8 & $-10.7,9.2$ \\
\hline \multicolumn{2}{|c|}{ Education to age $18 * 3-<5$ hours (age 11 ) } & 1.1 & $0.2,6.2$ & 1.4 & $-10.1,12.8$ \\
\hline \multicolumn{2}{|c|}{ Education to age $18 * 5$ hours or more (age 11 ) } & 0.8 & $0.1,5.1$ & -1.0 & $-13.6,11.7$ \\
\hline \multicolumn{2}{|c|}{ Education to age $16^{*} 1-<3$ hours (age 11 ) } & 0.7 & $0.2,1.9$ & -0.6 & $-7.3,6.2$ \\
\hline \multicolumn{2}{|c|}{ Education to age $16 * 3-<5$ hours (age 11 ) } & 0.8 & $0.3,2.7$ & 1.8 & $-6.4,10.0$ \\
\hline \multicolumn{2}{|c|}{ Education to age $16 * 5$ hours or more (age 11 ) } & 0.8 & $0.2,2.6$ & 1.9 & $-7.1,10.8$ \\
\hline \multicolumn{2}{|c|}{ No qualifications* $1-<3$ hours (age 11 ) } & 0.9 & $0.2,4.3$ & 1.4 & $-8.6,11.4$ \\
\hline \multicolumn{2}{|c|}{ No qualifications $* 3-<5$ hours (age 11 ) } & 1.2 & $0.2,6.0$ & 4.4 & $-6.9,15.7$ \\
\hline \multicolumn{2}{|c|}{ No qualifications*5 hours or more (age 11 ) } & 0.9 & $0.2,4.6$ & 2.4 & $-8.5,13.4$ \\
\hline
\end{tabular}

F-test of the cross-product terms on the risk ratio scale: $\mathrm{F}(9,1000)=0.13 ; \mathrm{p}=0.999$

F-test of the cross-product terms on the risk difference scale: $F(9,1000)=0.20 ; p=0.994$ 


\section{eAppendix 3. Causal diagrams and inverse probability weights}

eFigure A3.1. Causal diagram (Directed Acyclic Graph) of the mediation analysis: A=mother's education (in this diagram shown as if it were effectively randomized), $M_{t}=$ screen media exposure, $V=$ time fixed (baseline) confounders, $\mathrm{L}_{\mathrm{t}}=$ time-varying confounders, $\mathrm{Y}=$ childhood obesity, $\mathrm{U}=$ unmeasured confounders. $\mathrm{L}_{\mathrm{t}}$ is on the causal pathway $\mathrm{A} \rightarrow \mathrm{Y}$, but also a confounder in the relationship $\mathrm{M}_{\mathrm{t}} \rightarrow \mathrm{Y}$, which prohibits conventional adjustment for $\mathrm{L}_{\mathrm{t}}$.

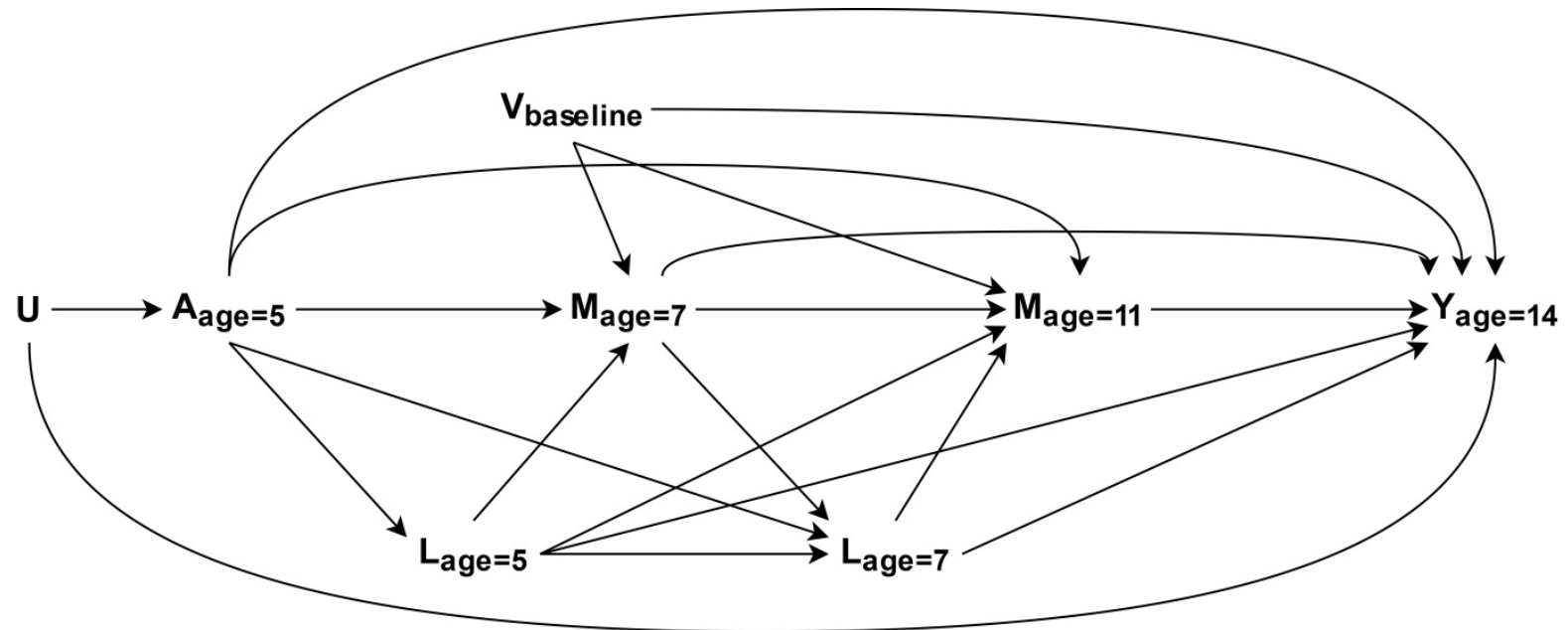

To adjust for (time-varying) mediator-outcome confounders that are itself affected by the exposure (see Figure A3.1), we fitted stabilized inverse-probability-weighted marginal structural models. ${ }^{12} 1315$ By using weights instead of conditioning on confounders, it is possible to effectively eliminate mediator-outcome confounding effects while still leaving the pathway from exposure to outcome intact (See Figure A3.2). In our case, we estimated stabilized inverse probability weights by calculating and multiplying the weights defined by equation (5). For each individual $i$ in the sample, the mediator weight at time $t$ is calculated by

(1) $\mathrm{w}^{\mathrm{M}}{ }_{\mathrm{i}}(\mathrm{t})=\frac{\mathrm{P}\left\{\mathrm{M}(\mathrm{t})=\mathrm{m}_{i}(\mathrm{t}) \mid \mathrm{a}_{i}, \mathrm{~m}_{i}(\mathrm{t}-1)\right\}}{\mathrm{P}\left\{\mathrm{M}(\mathrm{t})=\mathrm{m}_{i}(\mathrm{t}) \mid \mathrm{a}_{i}, \mathrm{~m}_{i}(\mathrm{t}-1), \mathrm{l}_{i}(t-1), \mathrm{v}_{i}\right\}}$

where $a_{i}, m_{i}(t), l_{i}(t)$, and $v_{i}$ are the actual values of the exposure, the mediator, the time-varying confounders, and the baseline confounders, respectively, for individual $i$. The numerator of $w^{M}{ }_{i}(t)$ is the probability of individual $i$ having the number of hours of screen media exposure that they actually had at time $t$, conditional on exposure and mediator history. The denominator of $w^{M}{ }_{i}(t)$ is the probability of individual $i$ having the number of hours of screen media exposure that they actually had at time $t$, conditional on exposure, mediator history, time-varying confounder history, and baseline confounders. The resulting overall weight $w^{M}{ }_{i}=w^{M}{ }_{i}(4) * w^{M}{ }_{i}(5)$ was used in the fitted regression models (3) and (4) to control for possible confounding. The coefficients from these regression models will give the counterfactual disparity measure of the unmediated association between mother's education and childhood obesity provided that exposure $A$ and measured confounders in vectors $V$ and $L$ suffice to control for confounding between screen media exposure and childhood obesity. Survey weights were incorporated into the marginal structural model by taking the product of $w^{M}{ }_{i}$ and the survey weights. ${ }^{33}$

Weights for the exposure were not estimated, because our aim was to estimate to what extent social inequalities in childhood obesity could be reduced if we were to intervene on screen media exposure (i.e. the counterfactual disparity measure), which does not require identification of a causal effect of 
education on childhood obesity. Consequently, we did not adjust for exposure-outcome confounders and exposure weights were not needed.

eTable A3.1. Distribution of the weights.

\begin{tabular}{lllll}
\hline Weights & Mean & SD & Range & IQR \\
\hline $\mathrm{W}^{\mathrm{M}}{ }_{\mathrm{i}}(4)$ & 1.0 & 0.2 & $0.3-3.7$ & $0.9-1.1$ \\
$\mathrm{~W}^{\mathrm{M}}{ }_{\mathrm{i}}(5)$ & 1.0 & 0.2 & $0.2-4.6$ & $0.9-1.1$ \\
$\mathrm{~W}^{\mathrm{M}}{ }_{\mathrm{i}}$ & 1.0 & 0.4 & $0.2-6.2$ & $0.8-1.1$ \\
\hline
\end{tabular}

eFigure A3.2. Causal diagram of the scenario encountered after applying the inverse probability weights: $A=$ =parental education, $\mathrm{M}_{\mathrm{t}}=$ screen media exposure (depicted with a box to indicate that they are conditioned on in the regression model), $\mathrm{V}=$ time fixed (baseline) confounders, $\mathrm{L}_{\mathrm{t}}=$ time-varying confounders, $\mathrm{Y}=$ childhood obesity, $\mathrm{U}=$ unmeasured confounders. The dashed lines depict the counterfactual disparity measure. By applying the weights several arrows are 'erased' (i.e. the effect of $\mathrm{V}$ and Lt on $\mathrm{Mt}$ ) and it becomes possible to estimate the combined magnitude of the dashed lines..

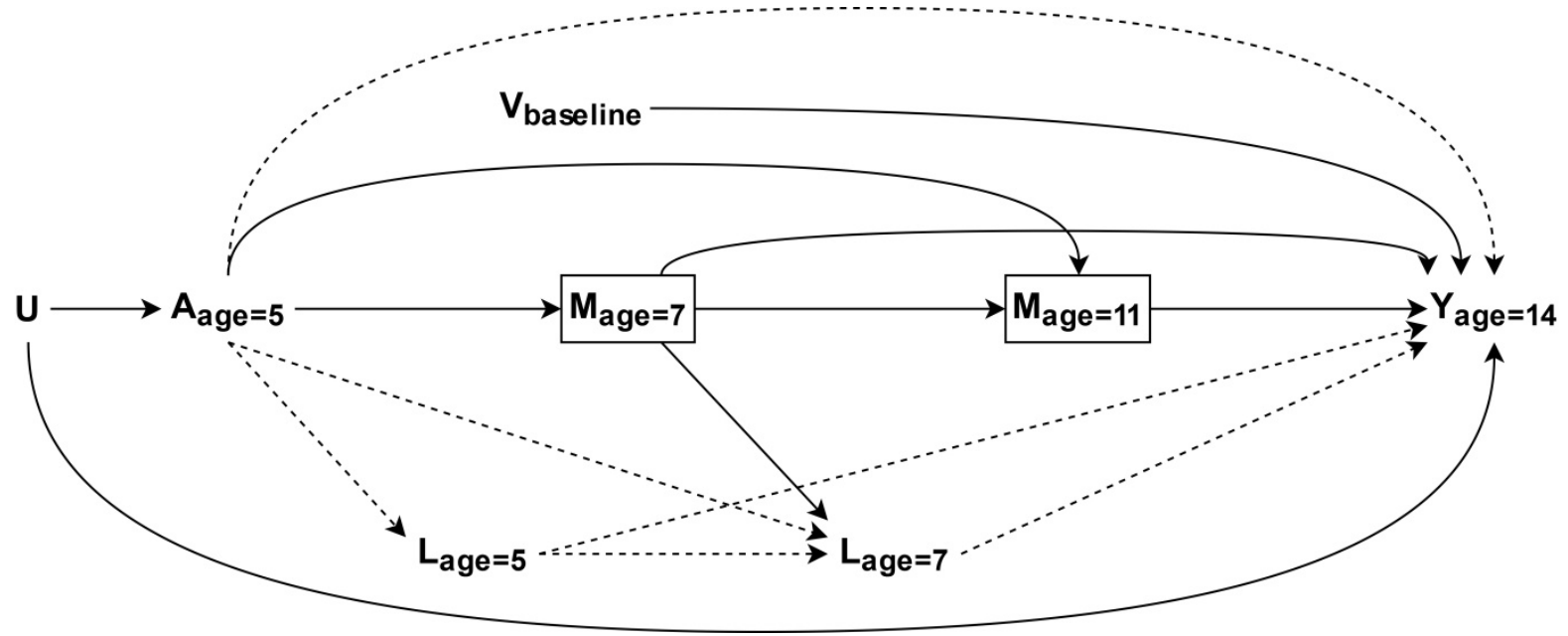


Annotated Stata code for inverse probability of treatment weighting of a marginal structural model to derive controlled direct effects using time-varying mediators

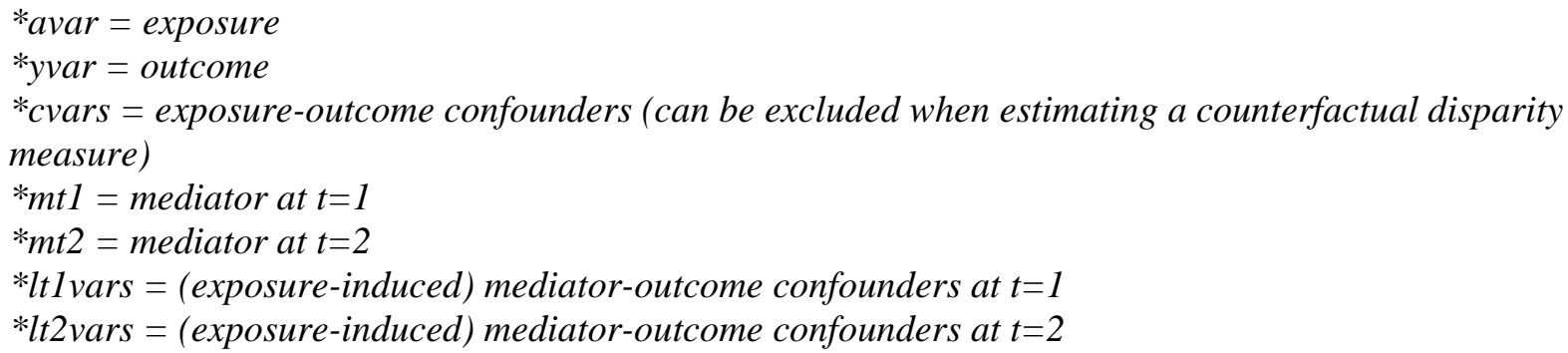

* calculate numerator for exposure weights (exposure has 4 categories)

mlogit avar, b(1)

foreach num of numlist $2 / 4\{$

predict xb_avar_n_'num', $x b$ equation(\# num')

gen p_avar_n_’num'=exp(xb_avar_n_'num')

\}

gen pred_avar_n=1/(1+p_avar_n_2+p_avar_n_3+p_avar_n_4 $)$ if avar==1

replace pred_avar_n=p_avar_n_2/(1+p_avar_n_2+p_avar_n_3+p_avar_n_4) if avar==2

replace pred_avar_n=p_avar_n_3/(1+p_avar_n_2+p_avar_n_3+p_avar_n_4 $)$ if avar==3

replace pred_avar_n=p_avar_n_4/(1+p_avar_n_2+p_avar_n_3+p_avar_n_4) if avar==4

* calculate denominator for exposure weights

mlogit avar cvars, b(1)

foreach num of numlist $2 / 4$ \{

predict xb_avar_d_'num', xb equation(\#`num')

gen p_avar_d_`num'=exp(xb_avar_d_'num')

\}

gen pred_avar_d=1/(1+p_avar_d_2+p_avar_d_3+p_avar_d_4 $)$ if avar==1

replace pred_avar_d=p_avar_d_2/(1+p_avar_d_2+p_avar_d_3+p_avar_d_4 $)$ if avar==2

replace pred_avar_d=p_avar_d_3/(1+p_avar_d_2+p_avar_d_3+p_avar_d_4) if avar==3

replace pred_avar_d=p_avar_d_4/(1+p_avar_d_2+p_avar_d_3+p_avar_d_4) if avar==4

* calculate exposure weights

gen ipwavar=pred_avar_n/pred_avar_d

* calculate numerator for mediator weights at time $\mathrm{t}=1$ (mediator has 4 categories; use dummy variables if covariates are categorical, e.g. in our example we include 3 dummies for avar) mlogit mt1 avar, b(1)

foreach num of numlist $2 / 4$ \{

predict xb_mt1_n_'num', xb equation(\#`num')

qui gen p_mt1_n_'num'=exp(xb_mt1_n_'num')

\}

gen pred_mt1_n=1/(1+p_mt1_n_2+p_mt1_n_3+p_mt1_n_4) if $\mathrm{mt} 1==1$

replace pred_mt1_n=p_mt1_n_2/(1+p_mt1_n_2+p_mt1_n_3+p_mt1_n_4) if $\mathrm{mt} 1==2$

replace pred_mt1_n=p_mt1_n_3/(1+p_mt1_n_2+p_mt1_n_3+p_mt1_n_4) if $\mathrm{mt} 1==3$

replace pred_mt1_n=p_mt1_n_4/(1+p_mt1_n_2+p_mt1_n_3+p_mt1_n_4) if $m t 1==4$ 
* calculate denominator for mediator weights at time $\mathrm{t}=1$

mlogit mt1 avar lt1vars cvars, b(1)

foreach num of numlist 2/4 \{

predict xb_mt1_d_'num', xb equation(\#`num')

gen p_mt1_d_`num'=exp(xb_mt1_d_`num')

\}

gen pred_mt1_d=1/(1+p_mt1_d_2+p_mt1_d_3+p_mt1_d_4 $)$ if $m t 1==1$

replace pred_mt1_d=p_mt1_d_2/(1+p_mt1_d_2+p_mt1_d_3+p_mt1_d_4) if $\mathrm{mt} 1==2$

replace pred_mt1_d=p_mt1_d_3/(1+p_mt1_d_2+p_mt1_d_3+p_mt1_d_4) if $\mathrm{mt} 1==3$

replace pred_mt1_d=p_mt1_d_4/(1+p_mt1_d_2+p_mt1_d_3+p_mt1_d_4) if $\mathrm{mt} 1==4$

* calculate mediator weights $\mathrm{t}=1$

gen ipwmt1=pred_mt1_n/pred_mt1_d

* calculate numerator for mediator weights at time $\mathrm{t}=2$

mlogit mt2 avar mt1, b(1)

foreach num of numlist $2 / 4$ \{

predict xb_mt2_n_'num', xb equation(\#`num')

gen p_mt2_n_`num'=exp(xb_mt2_n_'num')

\}

gen pred_mt2_n=1/(1+p_mt2_n_2+p_mt2_n_3+p_mt2_n_4) if $m t 2==1$

replace pred_mt2_n=p_mt2_n_2/(1+p_mt2_n_2+p_mt2_n_3+p_mt2_n_4) if $\mathrm{mt} 2==2$

replace pred_mt2_n=p_mt2_n_3/(1+p_mt2_n_2+p_mt2_n_3+p_mt2_n_4) if $\mathrm{mt} 2==3$

replace pred_mt2_n=p_mt2_n_4/(1+p_mt2_n_2+p_mt2_n_3+p_mt2_n_4) if mt2==4

* calculate denominator for mediator weights at time $\mathrm{t}=2$

mlogit mt2 avar mt1 lt1vars lt2vars cvars, b(1)

foreach num of numlist $2 / 4$ \{

predict xb_mt2_d_'num', xb equation(\#`num')

gen p_mt2_d_`num'=exp(xb_mt2_d_'num')

\}

gen pred_mt2_d=1/(1+p_mt2_d_2+p_mt2_d_3+p_mt2_d_4 $)$ if $m t 2==1$

replace pred_mt2_d=p_mt2_d_2/(1+p_mt2_d_2+p_mt2_d_3+p_mt2_d_4 $)$ if $m t 2==2$

replace pred_mt2_d=p_mt2_d_3/(1+p_mt2_d_2+p_mt2_d_3+p_mt2_d_4) if $\mathrm{mt} 2==3$

replace pred_mt2_d=p_mt2_d_4/(1+p_mt2_d_2+p_mt2_d_3+p_mt2_d_4) if $\mathrm{mt} 2==4$

* calculate mediator weights $\mathrm{t}=2$

gen ipwmt2=pred_mt2_n/pred_mt2_d

* calculate inverse probability weights (multiply by sample weight if applicable)

gen msmwgt=ipwavar*ipwmt $1 * i p w m t 2$

* Estimate controlled direct effect (include interaction terms if applicable; other specifications of the marginal structural model can also be considered)

glm yvar avar mt1 mt2 [pweight=msmwgt], fam(poisson) link(log) vce(robust) eform 
Bootstrapping to derive confidence intervals for 'percentage eliminated'

* Define a user-writter program

capture program drop CDE

program CDE, rclass

* Insert the total effect regression (use dummy variables if covariates are categorical, e.g. in our example we include 3 dummies for avar)

glm yvar avar cvars, fam(poisson) link(log) vce(robust)

matrix m_total=e(b)

scalar b_total1 $=$ m_total $[1,1]$

return scalar b_total1 $=$ m_total[1,1]

scalar b_total2=m_total[1,2]

return scalar b_total2 $=\mathrm{m} \_$total[ $[1,2]$

scalar b_total $3=$ m_total $[1,3]$

return scalar b_total3=m_total[1,3]

* Insert controlled direct effect regression (include interaction terms if applicable)

glm yvar avar mt1 mt2 [pweight=msmwgt], fam(poisson) link(log) vce(robust)

matrix m_direct $=\mathrm{e}(\mathrm{b})$

scalar b_direct1=m_direct $[1,1]$

return scalar b_direct $1=\mathrm{m} \_$direct $[1,1]$

scalar b_direct $2=$ m_direct $[1,2]$

return scalar b_direct2=m_direct[1,2]

scalar b_direct $3=m \_$direct $[1,3]$

return scalar b_direct3=m_direct[1,3]

return scalar RRp_elim1=(exp(b_total1)-exp(b_direct1) $) /(\exp ($ b_total1)-1)

return scalar RRp_elim2=(exp(b_total2)-exp(b_direct2) $) /(\exp ($ b_total2)-1)

return scalar RRp_elim3=(exp(b_total3)-exp(b_direct3) $) /\left(\exp \left(b \_t o t a l 3\right)-1\right)$

end

* Provide initial value of the random-number seed so estimates can be replicated at a later time. set seed 1234

* Request bootstrapped estimates of controlled direct effect and proportion eliminated (we also request percentile and bias-corrected bootstrap)

bootstrap PE1=r(RRp_elim1) PE2=r(RRp_elim2) PE3=r(RRp_elim3), reps(1000):CDE

estat boot, percentile bc 
Annotated Stata code for inverse probability of treatment weighting of a marginal structural model to derive controlled direct effects using time-varying mediators and multiple imputation to impute missing data

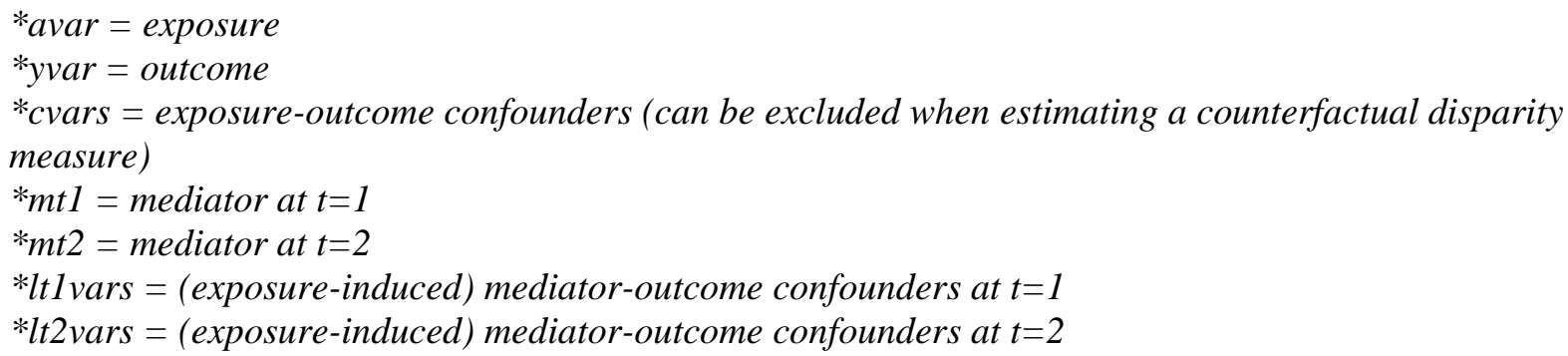

* calculate numerator for exposure weights (exposure has 4 categories)

mim, storebv: mlogit avar, b(1)

foreach num of numlist $1 / 3$ \{

predict xb_avar_n_'num', xb equation(\#num')

gen p_avar_n_'num'=exp(xb_avar_n_'num')

\}

gen pred_avar_n=1/(1+p_avar_n_1+p_avar_n_2+p_avar_n_3) if avar==1

replace pred_avar_n=p_avar_n_1/(1+p_avar_n_1+p_avar_n_2+p_avar_n_3) if avar==2

replace pred_avar_n=p_avar_n_2/(1+p_avar_n_1+p_avar_n_2+p_avar_n_3) if avar==3

replace pred_avar_n=p_avar_n_3/(1+p_avar_n_1+p_avar_n_2+p_avar_n_3) if avar==4

* calculate denominator for exposure weights

mim, storebv: mlogit avar cvars, b(1)

foreach num of numlist $1 / 3$ \{

predict xb_avar_d_`num', xb equation(\#`num')

gen p_avar_d_'num'=exp(xb_avar_d_'num')

\}

gen pred_avar_d=1/(1+p_avar_d_1+p_avar_d_2+p_avar_d_3 $)$ if avar==1

replace pred_avar_d=p_avar_d_1/(1+p_avar_d_1+p_avar_d_2+p_avar_d_3 $)$ if avar==2

replace pred_avar_d=p_avar_d_2/(1+p_avar_d_1+p_avar_d_2+p_avar_d_3) if avar==3

replace pred_avar_d=p_avar_d_3/(1+p_avar_d_1+p_avar_d_2+p_avar_d_3) if avar==4

* calculate exposure weights

gen ipwavar=pred_avar_n/pred_avar_d

* calculate numerator for mediator weights at time $\mathrm{t}=1$ (mediator has 4 categories; use dummy variables if covariates are categorical, e.g. in our example we include 3 dummies for avar) mim, storebv: mlogit mt1 avar, b(1)

foreach num of numlist $1 / 3$ \{

predict xb_mt1_n_'num', xb equation(\#`num')

qui gen p_mt1_n_'num'=exp(xb_mt1_n_'num')

\}

gen pred_mt1_n=1/(1+p_mt1_n_1+p_mt1_n_2+p_mt1_n_3) if mt1==1

replace pred_mt1_n=p_mt1_n_1/(1+p_mt1_n_1+p_mt1_n_2+p_mt1_n_3) if $\mathrm{mt1}==2$

replace pred_mt1_n=p_mt1_n_2/(1+p_mt1_n_1+p_mt1_n_2+p_mt1_n_3) if $\mathrm{mt} 1==3$

replace pred_mt1_n=p_mt1_n_3/(1+p_mt1_n_1+p_mt1_n_2+p_mt1_n_3) if $\mathrm{mt} 1==4$ 
* calculate denominator for mediator weights at time $\mathrm{t}=1$

mim, storebv: mlogit mt1 avar lt1vars cvars, b(1)

foreach num of numlist $1 / 3$ \{

predict xb_mt1_d_'num', xb equation(\#`num')

gen p_mt1_d_`num'=exp(xb_mt1_d_`num')

\}

gen pred_mt1_d=1/(1+p_mt1_d_1+p_mt1_d_2+p_mt1_d_3 $)$ if $m t 1==1$

replace pred_mt1_d=p_mt1_d_1/(1+p_mt1_d_1+p_mt1_d_2+p_mt1_d_3 $)$ if $\mathrm{mt} 1==2$

replace pred_mt1_d=p_mt1_d_2/(1+p_mt1_d_1+p_mt1_d_2+p_mt1_d_3) if $\mathrm{mt} 1==3$

replace pred_mt1_d=p_mt1_d_3/(1+p_mt1_d_1+p_mt1_d_2+p_mt1_d_3 $)$ if $m t 1==4$

* calculate mediator weights $\mathrm{t}=1$

gen ipwmt1=pred_mt1_n/pred_mt1_d

* calculate numerator for mediator weights at time $\mathrm{t}=2$

mim, storebv: mlogit mt2 avar mt1, b(1)

foreach num of numlist $1 / 3$ \{

predict xb_mt2_n_'num', xb equation(\#`num')

gen p_mt2_n_`num'=exp(xb_mt2_n_'num')

\}

gen pred_mt2_n=1/(1+p_mt2_n_1+p_mt2_n_2+p_mt2_n_3) if $\mathrm{mt} 2==1$

replace pred_mt2_n=p_mt2_n_1/(1+p_mt2_n_1+p_mt2_n_2+p_mt2_n_3) if $\mathrm{mt} 2==2$

replace pred_mt2_n=p_mt2_n_2/(1+p_mt2_n_1+p_mt2_n_2+p_mt2_n_3) if $\mathrm{mt} 2==3$

replace pred_mt2_n=p_mt2_n_3/(1+p_mt2_n_1+p_mt2_n_2+p_mt2_n_3) if mt2==4

* calculate denominator for mediator weights at time $\mathrm{t}=2$

mim, storebv: mlogit mt2 avar mt1 lt1vars lt2vars cvars, b(1)

foreach num of numlist $1 / 3$ \{

predict xb_mt2_d_'num', xb equation(\#`num')

gen p_mt2_d_`num'=exp(xb_mt2_d_'num')

\}

gen pred_mt2_d=1/(1+p_mt2_d_1+p_mt2_d_2+p_mt2_d_3 $)$ if $\mathrm{mt} 2==1$

replace pred_mt2_d=p_mt2_d_1/(1+p_mt2_d_1+p_mt2_d_2+p_mt2_d_3 $)$ if $m t 2==2$

replace pred_mt2_d=p_mt2_d_2/(1+p_mt2_d_1+p_mt2_d_2+p_mt2_d_3) if $\mathrm{mt} 2==3$

replace pred_mt2_d=p_mt2_d_3/(1+p_mt2_d_1+p_mt2_d_2+p_mt2_d_3) if mt2==4

* calculate mediator weights $\mathrm{t}=2$

gen ipwmt2=pred_mt2_n/pred_mt2_d

* calculate inverse probability weights (multiply by sample weight if applicable)

gen msmwgt=ipwavar*ipwmt1*ipwmt2

* Estimate controlled direct effect (include interaction terms if applicable; other specifications of the marginal structural model can also be considered)

mim, storebv: glm yvar avar mt1 mt2 [pweight=msmwgt], fam(poisson) link(log) vce(robust) eform 
Bootstrapping to derive confidence intervals for 'percentage eliminated'

* Define a user-writter program capture program drop CDE_MI program CDE_MI, rclass

* Preserve the data preserve

* Insert equation to impute missing data. We request 20 imputed datasets ice yvar avar mt2 mt1 cvars lt1vars lt2vars MIvars, saving("filelocation\nameofdataset.dta", replace) $\mathrm{m}(20)$

use " filelocation\nameofdataset.dta", clear

* calculate numerator for exposure weights (exposure has 4 categories)

mim, storebv: mlogit avar, b(1)

foreach num of numlist $1 / 3$ \{

predict xb_avar_n_'num', $x b$ equation(\#num')

gen p_avar_n_'num'=exp(xb_avar_n_'num')

\}

gen pred_avar_n=1/(1+p_avar_n_1+p_avar_n_2+p_avar_n_3) if avar==1

replace pred_avar_n=p_avar_n_1/(1+p_avar_n_1+p_avar_n_2+p_avar_n_3) if avar==2

replace pred_avar_n=p_avar_n_2/(1+p_avar_n_1+p_avar_n_2+p_avar_n_3) if avar==3

replace pred_avar_n=p_avar_n_3/(1+p_avar_n_1+p_avar_n_2+p_avar_n_3) if avar==4

* calculate denominator for exposure weights

mim, storebv: mlogit avar cvars, b(1)

foreach num of numlist $1 / 3$ \{

predict xb_avar_d_`num', xb equation(\#`num')

gen p_avar_d_’num'=exp(xb_avar_d_'num')

\}

gen pred_avar_d=1/(1+p_avar_d_1+p_avar_d_2+p_avar_d_3 $)$ if avar==1

replace pred_avar_d=p_avar_d_1/(1+p_avar_d_1+p_avar_d_2+p_avar_d_3 $)$ if avar==2

replace pred_avar_d=p_avar_d_2/(1+p_avar_d_1+p_avar_d_2+p_avar_d_3) if avar==3

replace pred_avar_d=p_avar_d_3/(1+p_avar_d_1+p_avar_d_2+p_avar_d_3) if avar==4

* calculate exposure weights

gen ipwavar=pred_avar_n/pred_avar_d

* calculate numerator for mediator weights at time $\mathrm{t}=1$ (mediator has 4 categories; use dummy variables if covariates are categorical, e.g. in our example we include 3 dummies for avar) mim, storebv: mlogit mt1 avar, b(1)

foreach num of numlist $1 / 3$ \{

predict xb_mt1_n_`num', xb equation(\#`num')

qui gen p_mt1_n_'num'=exp(xb_mt1_n_'num')

\}

gen pred_mt1_n=1/(1+p_mt1_n_1+p_mt1_n_2+p_mt1_n_3) if mt1==1

replace pred_mt1_n=p_mt1_n_1/(1+p_mt1_n_1+p_mt1_n_2+p_mt1_n_3) if mt1==2 
replace pred_mt1_n=p_mt1_n_2/(1+p_mt1_n_1+p_mt1_n_2+p_mt1_n_3) if $\mathrm{mt1}==3$ replace pred_mt1_n=p_mt1_n_3/(1+p_mt1_n_1+p_mt1_n_2+p_mt1_n_3) if $\mathrm{mt1}==4$

* calculate denominator for mediator weights at time $\mathrm{t}=1$ mim, storebv: mlogit mt1 avar lt1vars cvars, b(1)

foreach num of numlist $1 / 3$ \{ predict xb_mt1_d_'num', xb equation(\#`num') gen p_mt1_d_'num'=exp(xb_mt1_d_'num') \} gen pred_mt1_d=1/(1+p_mt1_d_1+p_mt1_d_2+p_mt1_d_3 $)$ if $m t 1==1$ replace pred_mt1_d=p_mt1_d_1/(1+p_mt1_d_1+p_mt1_d_2+p_mt1_d_3) if $\mathrm{mt} 1==2$ replace pred_mt1_d=p_mt1_d_2/(1+p_mt1_d_1+p_mt1_d_2+p_mt1_d_3 $)$ if $\mathrm{mt} 1==3$ replace pred_mt1_d=p_mt1_d_3/(1+p_mt1_d_1+p_mt1_d_2+p_mt1_d_3) if $\mathrm{mt} 1==4$

* calculate mediator weights $\mathrm{t}=1$

gen ipwmt1=pred_mt1_n/pred_mt1_d

* calculate numerator for mediator weights at time $\mathrm{t}=2$

mim, storebv: mlogit mt2 avar mt1, b(1)

foreach num of numlist $1 / 3$ \{

predict xb_mt2_n_'num', xb equation(\#`num')

gen p_mt2_n_'num'=exp(xb_mt2_n_'num')

\}

gen pred_mt2_n=1/(1+p_mt2_n_1+p_mt2_n_2+p_mt2_n_3) if $m t 2==1$

replace pred_mt2_n=p_mt2_n_1/(1+p_mt2_n_1+p_mt2_n_2+p_mt2_n_3) if $\mathrm{mt} 2==2$ replace pred_mt2_n=p_mt2_n_2/(1+p_mt2_n_1+p_mt2_n_2+p_mt2_n_3) if $\mathrm{mt} 2==3$ replace pred_mt2_n=p_mt2_n_3/(1+p_mt2_n_1+p_mt2_n_2+p_mt2_n_3) if $\mathrm{mt} 2==4$

* calculate denominator for mediator weights at time $\mathrm{t}=2$

mim, storebv: mlogit mt2 avar mt1 lt1vars lt2vars cvars, b(1)

foreach num of numlist $1 / 3$ \{

predict xb_mt2_d_'num', xb equation(\#`num')

gen p_mt2_d_`num'=exp(xb_mt2_d_'num')

\}

gen pred_mt2_d=1/(1+p_mt2_d_1+p_mt2_d_2+p_mt2_d_3 $)$ if $m t 2==1$

replace pred_mt2_d=p_mt2_d_1/(1+p_mt2_d_1 + p_mt2_d_2+p_mt2_d_3 $)$ if $\mathrm{mt} 2==2$

replace pred_mt2_d=p_mt2_d_2/(1+p_mt2_d_1+p_mt2_d_2+p_mt2_d_3 $)$ if $\mathrm{mt} 2==3$

replace pred_mt2_d=p_mt2_d_3/(1+p_mt2_d_1+p_mt2_d_2+p_mt2_d_3) if mt2==4

* calculate mediator weights $\mathrm{t}=2$

gen ipwmt2=pred_mt2_n/pred_mt2_d

* calculate inverse probability weights (multiply by sample weight if applicable) gen msmwgt=ipwavar*ipwmt1*ipwmt2 
* Insert the total effect regression (use dummy variables if covariates are categorical, e.g. in our example we include 3 dummies for avar)

mim, storebv: glm yvar avar cvars, fam(poisson) link(log) vce(robust)

matrix m_total=e(b)

scalar b_total1=m_total[1,1]

return scalar b_total1 $=$ m_total[ $[1,1]$

scalar b_total2=m_total[1,2]

return scalar b_total2=m_total[1,2]

scalar b_total3=m_total[1,3]

return scalar b_total3=m_total[1,3]

* Insert controlled direct effect regression (include interaction terms if applicable)

mim, storebv: glm yvar avar mt1 mt2 [pweight=msmwgt], fam(poisson) link(log) vce(robust)

matrix m_direct $=\mathrm{e}(\mathrm{b})$

scalar b_direct1=m_direct[1,1]

return scalar b_direct1=m_direct[1,1]

scalar b_direct $2=$ m_direct $[1,2]$

return scalar b_direct $2=m \_$direct $[1,2]$

scalar b_direct3=m_direct $[1,3]$

return scalar b_direct3=m_direct $[1,3]$

return scalar RRp_elim1=(exp(b_total1)-exp(b_direct1) $) /\left(\exp \left(b \_t o t a l 1\right)-1\right)$

return scalar RRp_elim2 $=\left(\exp \left(\mathrm{b} \_t\right.\right.$ total2)-exp(b_direct2) $) /\left(\exp \left(\mathrm{b} \_t o t a l 2\right)-1\right)$

return scalar RRp_elim3=(exp(b_total3)-exp(b_direct3) $) /\left(\exp \left(b \_t o t a l 3\right)-1\right)$

end

* Provide initial value of the random-number seed so estimates can be replicated at a later time. set seed 1234

* Request bootstrapped estimates of controlled direct effect and proportion eliminated (we also request percentile and bias-corrected bootstrap)

bootstrap PE1=r(RRp_elim1) PE2=r(RRp_elim2) PE3=r(RRp_elim3), reps(1000):CDE_MI

estat boot, percentile bc 
eAppendix 4. Sensitivity analyses

eTable A4.1. Reduction in relative inequalities in childhood obesity if educational differences in screen media exposure were eliminated, using UK90 obesity cut-offs.

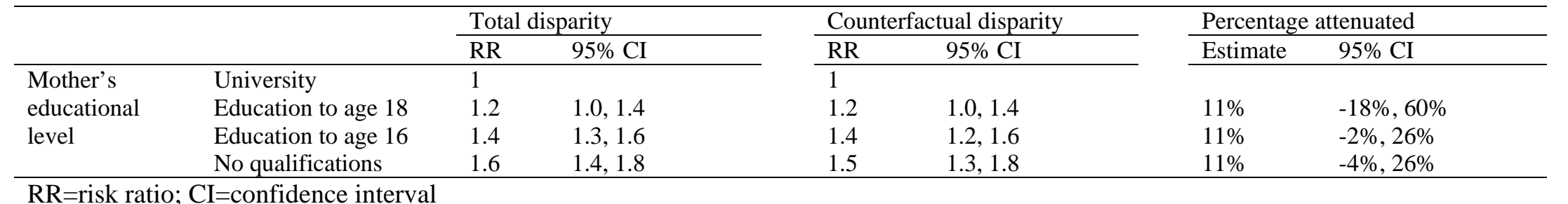

$\mathrm{RR}=$ risk ratio; $\mathrm{CI}=$ confidence interval

eTable A4.2. Reduction in absolute inequalities in childhood obesity if educational differences in screen media exposure were eliminated, using UK90 obesity cut-offs.

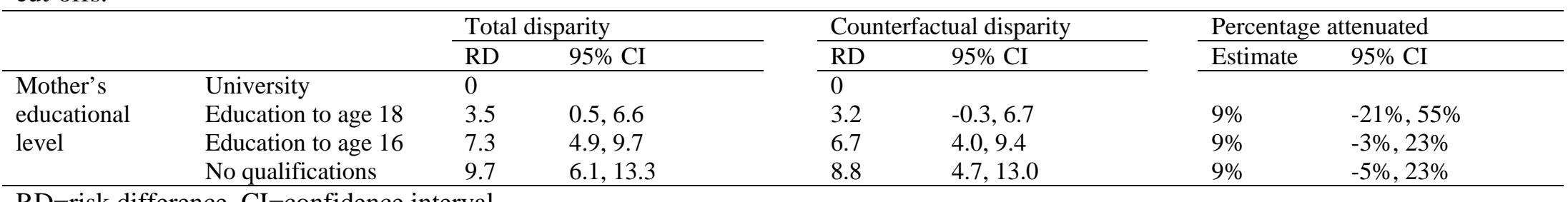

$\mathrm{RD}=$ risk difference, $\mathrm{CI}=$ confidence interval 
eTable A4.3. Reduction in relative inequalities in childhood obesity if educational differences in screen media exposure were eliminated, using highest parental educational level.

\begin{tabular}{|c|c|c|c|c|c|c|c|}
\hline & & \multicolumn{2}{|c|}{ Total disparity } & \multicolumn{2}{|c|}{ Counterfactual disparity } & \multicolumn{2}{|c|}{ Percentage attenuated } \\
\hline & & RR & 95\% CI & RR & $95 \%$ CI & Estimate & 95\% CI \\
\hline Highest & University & 1 & & 1 & & & \\
\hline parental & Education to age 18 & 1.5 & $1.2,2.0$ & 1.4 & $1.1,1.9$ & $16 \%$ & $-3 \%, 38 \%$ \\
\hline educational & Education to age 16 & 1.8 & $1.5,2.2$ & 1.7 & $1.4,2.2$ & $8 \%$ & $-5 \%, 22 \%$ \\
\hline level & No qualifications & 2.0 & $1.5,2.6$ & 1.9 & $1.4,2.5$ & $9 \%$ & $-10 \%, 26 \%$ \\
\hline
\end{tabular}

$\mathrm{RR}=$ risk ratio; $\mathrm{CI}=$ confidence interval

eTable A4.4. Reduction in absolute inequalities in childhood obesity if educational differences in screen media exposure were eliminated, using highest parental educational level.

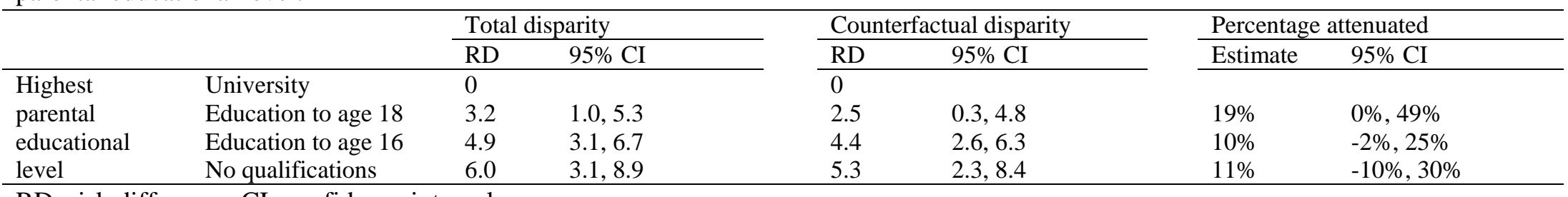

$\mathrm{RD}=$ risk difference, $\mathrm{CI}=$ confidence interval 
eTable A4.5. Reduction in relative inequalities in childhood obesity if income differences in screen media exposure were eliminated.

\begin{tabular}{|c|c|c|c|c|c|c|c|}
\hline & & \multicolumn{2}{|c|}{ Total disparity } & \multicolumn{2}{|c|}{ Counterfactual disparity } & \multicolumn{2}{|c|}{ Percentage attenuated } \\
\hline & & $\mathrm{RR}$ & $95 \% \mathrm{CI}$ & RR & $95 \% \mathrm{CI}$ & Estimate & $95 \% \mathrm{CI}$ \\
\hline Household & Highest (1) & 1 & & 1 & & & \\
\hline \multirow[t]{3}{*}{ income } & 2 & 1.2 & $0.9,1.6$ & 1.1 & $0.8,1.5$ & $43 \%$ & $-38 \%, 525 \%$ \\
\hline & 3 & 2.0 & $1.5,2.6$ & 1.8 & $1.3,2.4$ & $19 \%$ & $1 \%, 36 \%$ \\
\hline & Lowest (4) & 2.2 & $1.7,2.8$ & 2.0 & $1.5,2.6$ & $17 \%$ & $1 \%, 32 \%$ \\
\hline
\end{tabular}

$\mathrm{RR}=$ risk ratio; $\mathrm{CI}=$ confidence interval

eTable A4.6. Reduction in absolute inequalities in childhood obesity if income differences in screen media exposure were eliminated.

\begin{tabular}{|c|c|c|c|c|c|c|c|}
\hline & & \multicolumn{2}{|c|}{ Total disparity } & \multicolumn{2}{|c|}{ Counterfactual disparity } & \multicolumn{2}{|c|}{ Percentage attenuated } \\
\hline & & $\mathrm{RD}$ & $95 \% \mathrm{CI}$ & $\mathrm{RD}$ & $95 \% \mathrm{CI}$ & Estimate & $95 \% \mathrm{CI}$ \\
\hline Household & Highest (1) & 0 & & 0 & & & \\
\hline \multirow[t]{3}{*}{ income } & 2 & 1.3 & $-0.4,2.9$ & 0.7 & $-1.1,2.5$ & $47 \%$ & $-29 \%, 602 \%$ \\
\hline & 3 & 5.2 & $3.2,7.2$ & 4.2 & $1.8,6.6$ & $19 \%$ & $3 \%, 42 \%$ \\
\hline & Lowest (4) & 6.4 & $4.4,8.4$ & 5.4 & $3.2,7.6$ & $16 \%$ & $3 \%, 33 \%$ \\
\hline
\end{tabular}

$\mathrm{RD}=$ risk difference, $\mathrm{CI}=$ confidence interval 
eTable A4.7. Reduction in relative inequalities in childhood obesity if educational differences in television viewing were eliminated.

\begin{tabular}{|c|c|c|c|c|c|c|c|}
\hline & & \multicolumn{2}{|c|}{ Total disparity } & \multicolumn{2}{|c|}{ Counterfactual disparity } & \multicolumn{2}{|c|}{ Percentage attenuated } \\
\hline & & RR & $95 \% \mathrm{CI}$ & RR & $95 \% \mathrm{CI}$ & Estimate & $95 \% \mathrm{CI}$ \\
\hline \multirow{4}{*}{$\begin{array}{l}\text { Mother's } \\
\text { educational } \\
\text { level }\end{array}$} & University & 1 & & 1 & & & \\
\hline & Education to age 18 & 1.3 & $1.0,1.7$ & 1.2 & $0.9,1.6$ & $26 \%$ & $-46 \%, 192 \%$ \\
\hline & Education to age 16 & 1.9 & $1.5,2.3$ & 1.7 & $1.4,2.2$ & $15 \%$ & $-1 \%, 32 \%$ \\
\hline & No qualifications & 2.0 & $1.5,2.5$ & 1.8 & $1.3,2.4$ & $17 \%$ & $-6 \%, 41 \%$ \\
\hline
\end{tabular}

$\mathrm{RR}=$ risk ratio; $\mathrm{CI}=$ confidence interval

eTable A4.8. Reduction in absolute inequalities in childhood obesity if educational differences in television viewing were eliminated.

\begin{tabular}{|c|c|c|c|c|c|c|c|}
\hline & & \multicolumn{2}{|c|}{ Total disparity } & \multicolumn{2}{|c|}{ Counterfactual disparity } & \multicolumn{2}{|c|}{ Percentage attenuated } \\
\hline & & $\mathrm{RD}$ & $95 \%$ CI & $\mathrm{RD}$ & $95 \%$ CI & Estimate & $95 \% \mathrm{CI}$ \\
\hline \multirow{4}{*}{$\begin{array}{l}\text { Mother's } \\
\text { educational } \\
\text { level }\end{array}$} & University & 0 & & 0 & & & \\
\hline & Education to age 18 & 1.6 & $-0.4,3.6$ & 1.3 & $-0.9,3.4$ & $21 \%$ & $-55 \%, 173 \%$ \\
\hline & Education to age 16 & 5.1 & $3.4,6.7$ & 4.5 & $2.5,6.5$ & $11 \%$ & $-2 \%, 25 \%$ \\
\hline & No qualifications & 5.6 & $3.1,8.1$ & 4.9 & $2.0,7.8$ & $12 \%$ & $-9 \%, 35 \%$ \\
\hline
\end{tabular}

$\mathrm{RD}=$ risk difference, $\mathrm{CI}=$ confidence interval 
eTable A4.9. Reduction in relative inequalities in childhood obesity if educational differences in computer use were eliminated.

\begin{tabular}{|c|c|c|c|c|c|c|c|}
\hline & & \multicolumn{2}{|c|}{ Total disparity } & \multicolumn{2}{|c|}{ Counterfactual disparity } & \multicolumn{2}{|c|}{ Percentage attenuated } \\
\hline & & $\mathrm{RR}$ & $95 \% \mathrm{CI}$ & RR & $95 \% \mathrm{CI}$ & Estimate & $95 \% \mathrm{CI}$ \\
\hline \multirow{4}{*}{$\begin{array}{l}\text { Mother's } \\
\text { educational } \\
\text { level }\end{array}$} & University & 1 & & 1 & & & \\
\hline & Education to age 18 & 1.3 & $1.0,1.7$ & 1.1 & $0.8,1.5$ & $71 \%$ & $11 \%, 475 \%$ \\
\hline & Education to age 16 & 1.9 & $1.5,2.3$ & 1.7 & $1.4,2.2$ & $16 \%$ & $-7 \%, 35 \%$ \\
\hline & No qualifications & 2.0 & $1.5,2.5$ & 1.8 & $1.3,2.5$ & $13 \%$ & $-14 \%, 39 \%$ \\
\hline
\end{tabular}

$\mathrm{RR}=$ risk ratio; $\mathrm{CI}=$ confidence interval

eTable A4.10. Reduction in absolute inequalities in childhood obesity if educational differences in computer use were eliminated.

\begin{tabular}{|c|c|c|c|c|c|c|c|}
\hline & & \multicolumn{2}{|c|}{ Total disparity } & \multicolumn{2}{|c|}{ Counterfactual disparity } & \multicolumn{2}{|c|}{ Percentage attenuated } \\
\hline & & $\mathrm{RD}$ & $95 \%$ CI & $\mathrm{RD}$ & $95 \%$ CI & Estimate & $95 \% \mathrm{CI}$ \\
\hline \multirow{4}{*}{$\begin{array}{l}\text { Mother's } \\
\text { educational } \\
\text { level }\end{array}$} & University & 0 & & 0 & & & \\
\hline & Education to age 18 & 1.6 & $-0.4,3.6$ & 0.5 & $-1.6,2.6$ & $72 \%$ & $20 \%, 576 \%$ \\
\hline & Education to age 16 & 5.1 & $3.4,6.7$ & 4.6 & $2.6,6.7$ & $9 \%$ & $-10 \%, 26 \%$ \\
\hline & No qualifications & 5.6 & $3.1,8.1$ & 5.3 & $2.2,8.3$ & $6 \%$ & $-20 \%, 29 \%$ \\
\hline
\end{tabular}

$\mathrm{RD}=$ risk difference, $\mathrm{CI}=$ confidence interval 
eTable A4.11. Reduction in relative inequalities in childhood obesity if educational differences in screen media exposure were eliminated, without using imputed data for exposure and outcome $(n=9,749)$.

\begin{tabular}{|c|c|c|c|c|c|c|c|}
\hline & & \multicolumn{2}{|c|}{ Total disparity } & \multicolumn{2}{|c|}{ Counterfactual disparity } & \multicolumn{2}{|c|}{ Percentage attenuated } \\
\hline & & RR & $95 \%$ CI & RR & 95\% CI & Estimate & $95 \%$ CI \\
\hline \multirow{4}{*}{$\begin{array}{l}\text { Mother's } \\
\text { educational } \\
\text { level }\end{array}$} & University & 1 & & 1 & & & \\
\hline & Education to age 18 & 1.4 & $1.0,1.8$ & 1.3 & $0.9,1.7$ & $32 \%$ & $-10 \%, 162 \%$ \\
\hline & Education to age 16 & 2.1 & $1.7,2.6$ & 1.9 & $1.5,2.5$ & $16 \%$ & $0 \%, 31 \%$ \\
\hline & No qualifications & 2.1 & $1.6,2.8$ & 1.9 & $1.3,2.6$ & $21 \%$ & $-5 \%, 48 \%$ \\
\hline
\end{tabular}

$\mathrm{RR}=$ risk ratio; $\mathrm{CI}=$ confidence interval

eTable A4.12. Reduction in absolute inequalities in childhood obesity if educational differences in screen media exposure were eliminated, without using imputed data for exposure and outcome $(n=9,479)$.

\begin{tabular}{|c|c|c|c|c|c|c|c|}
\hline & \multicolumn{2}{|c|}{ Total disparity } & \multicolumn{2}{|c|}{ Counterfactual disparity } & \multicolumn{2}{|c|}{ Percentage attenuated } \\
\hline & & $\mathrm{RD}$ & $95 \% \mathrm{CI}$ & $\mathrm{RD}$ & $95 \%$ CI & Estimate & $95 \%$ CI \\
\hline \multirow{4}{*}{$\begin{array}{l}\text { Mother's } \\
\text { educational } \\
\text { level }\end{array}$} & University & 0 & & 0 & & & \\
\hline & Education to age 18 & 1.8 & $0.0,3.5$ & 1.2 & $-0.5,2.9$ & $32 \%$ & $-26 \%, 211 \%$ \\
\hline & Education to age 16 & 5.2 & $3.6,6.8$ & 4.4 & $2.6,6.1$ & $16 \%$ & $1 \%, 32 \%$ \\
\hline & No qualifications & 5.1 & $2.7,7.5$ & 4.0 & $1.4,6.6$ & $22 \%$ & $-8 \%, 51 \%$ \\
\hline
\end{tabular}

$\mathrm{RD}=$ risk difference, $\mathrm{CI}=$ confidence interval 